\title{
Analog Direct-Modulation Behavior of Semiconductor Laser Transmitters Using Optical FM Demodulation
}

\author{
Gnitabouré Yabre, Member, IEEE
}

\begin{abstract}
In this paper, we report a theoretical investigation of the analog modulation performance of a semiconductor laser transmitter which employs the direct optical FM demodulation. This analysis is based on the rate equations in which Langevin noise functions are included. The optical FM response has firstly been derived using a chirp-power relation deduced from the rate equations. The chirp-to-power ratio (CPR) has secondly been computed and illustrated showing the effect of the novel damping parameter phenomenologically introduced in the rate equation for the optical phase. The curves show that the conventional CPR can be notably reduced using FM demodulation. Beside this, the comparison of the FM responses with prior studies show that our model may be more appropriate to describe optical FM behavior of many types of single-mode lasers. The relative-output noise (RON) at the transmitter has also been determined and subsequently used to calculate the carrier-to-noise ratio (CNR) available. It is shown that the transmitter inherent noise would significantly degrade as a result of the conversion of the optical frequency noise into intensity noise. Consequently, intrinsic noise is expected practically to contribute the most to CNR at high powers. This feature can be more easily appreciated through curves illustrating CNR and is in contrast to previous studies in which the receiver shot noise was assumed to be the main noise term.
\end{abstract}

Index Terms-Direct modulation, Mach-Zehnder interferometer (MZI), noise, nonlinear distortion, semiconductor lasers.

\section{INTRODUCTION}

O PTICAL fibers enable the transmission of information over long distances using either digital or analog type of modulation. Because of noise performance and general tendency of the technology toward digital modulation, this transmission format has dominated the fiber-optic systems development. However, taking advantage of simpler hardware configuration, analog type of modulation has emerged as an important area for applications of semiconductor lasers. These applications range from broadband microwave links for satellite communications to cable-television applications that operate below $1 \mathrm{GHz}$. Both FM or AM type of modulation can be used to carry information over the optical fiber. Generally,

Manuscript received May 21, 1997; revised November 21, 1997. This work was conducted within the framework of the Dutch Research Project IOP Electro-Optics in the COBRA Research Institute, Eindhoven University of Technology, The Netherlands.

The author is with COBRA, Interuniversity Research Institute, Eindhoven University of Technology, 5600 MB Eindhoven, The Netherlands.

Publisher Item Identifier S 0733-8724(98)01897-0. except in star-type local area networks where there is one only subcarrier per laser, many subcarriers from different channels are electrically combined (subcarrier multiplexing, SCM) and simultaneously transmitted on a single optical carrier. SCM technique allows for cost savings by sharing the same transmitter among several subscribers.

Unfortunately, SCM systems suffer from nonlinear distortion and noise that occur during the conversion process of the analog signals from current to light power, especially when a great number of channels modulate the laser. Inherent distortion originates mainly from three kinds of factors whose relative importance depends on the laser operation conditions. In the low-modulation frequency region, nonlinear distortion arises from the imperfect linearity of the static power-current characteristic, especially if the laser is biased high above threshold (static distortion) [1]. Another source of distortion, generally providing the dominant contribution, is the nonlinear coupling between photons and electrons inside the laser cavity. Because this distortion is frequency dependent and is responsible for relaxation osillation, it is denoted resonance distortion [2], [3]. The third mechanism of intrinsic distortion generation is tightly linked to the great number of channels that may be simultaneously transmitted. These subcarriers infrequently add themselves in phase at the receiver photodetecor causing random excursions of the drive current to values below threshold, for which the optical power falls at zero. This results in an extra distortion in the demodulated signal referred to as clipping distortion [4], [5].

An attempt has been made to reduce the effect of harmonics and intermodulation products using predistortion or feedforward compenation techniques [6], [7]. But, aside from their limited efficiency particularly at high modulation frequencies, these methods are not too easy to implement. Another alternative has been recently suggested [8] and theoretically demonstrated using the rate equations [9], [10]. It consists of converting the direct optical FM (also called frequency chirping) into IM signal by means of a Mach-Zehnder interferometer (MZI). Even though the analysis carried out in [10] deals with a two-tone modulation of the laser diode, the results allow one to expect a significant reduction of composite second order (CSO) and composite triple beat (CTB) in SCM systems. The MZI-based chirp demodulation process results in an important gain so that the laser needs lower current for the same optical modulation depth (OMD). This is the main reason 
behind reduction in resonance distortion. Moreover, since the laser needs not be modulated as much, static and clipping distortions can also be expected to reduce.

Practically speaking, optical FM demodulation can be achieved either at the transmitter or at the terminal receiver before direct detection. Installation of an FM-to-IM scheme at the customer's premises will enevitably increase his equipment cost. We believe that the former solution would be economically more justified because an optical transmitter, generally, serves many users on the network and any additional cost at the common transmission equipment can be shared among all, resulting in a nonsignificant extra contribution per subscriber.

However, the improvement in linearity expected in chirpdemodulation-based systems can be somewhat misleading in that nonlinear distortion is not the only technical criterion even though this characteristic is weighted heavily in deciding the performance of an SCM system. As mentioned above, in addition to $\mathrm{CTB}$ and $\mathrm{CSO}$, noise from the laser and receiver may be a severe limiting factor and is an important parameter. In classical direct-detection systems, the receiver shot and thermal noise as well as the laser relative-intensity noise (RIN) mainly contribute to the carrier-to-noise ratio (CNR) available at the subscriber's premises. Unfortunately, an FM-to-IM interferometer that demodulates optical FM, will additionally convert the optical frequency noise into IM noise resulting in RIN degradation. Therefore, the more useful benefits achieved using an MZI must be examined by evaluating the noise characteristics, so as the performance of an analog modulation system is a balance between noise and nonlinear distortion.

Another factor that can impair the performance of a fiberoptic link is the spectral linewidth broadening. Although this phenomenon has been often ignored when assessing system performance, it can affect the transmission quality of a singlemode fiber in combination with chromatic dispersion. An important parameter used to evaluate this effect is the chirpto-power ratio (CPR) defined as the ratio between optical FM and direct-intensity modulation efficiencies [11]. The CPR is is a convenient measure of FM because it gives the optical frequency shift for a given small-signal modulation power. Fortunately, an FM-to-IM interferometer can be expected to enhance the relative amplitude of the output signal resulting in an improved CPR characteristic.

In this paper, a proper expression of the CPR has been computed and illustrated. The study of RON and CNR has also been carried out. The curves are compared to the conventional transmission case showing higher levels of RON due to the extra conversion of the optical frequency noise into intensity noise. Consequently, the CNR is found to be mostly impaired by $\mathrm{RON}$ at large received powers. This result is in contrast with that forseen a few years ago [8] assuming the receiver shot noise to be the main contributor to CNR. Since the calculations carried out in [8] was based only on assumption and not on mathematical proof, we believe that our analysis is more appropriate as design guideline. This work completes the previous studies presented in [9], [10] investigating the transmitter nonlinear behavior. Using the present results together with [9] and [10], the actual improvement expected in SCM systems employing either conventional transmission or chirp demodulation technique can be properly weighted.

\section{THEORY}

In a strict sense, the description of laser diodes with spontaneous emission noise inclusion requires a quantum-mechanical formulation of the rate equations, because of the quantum nature of the emission process. Here we use the simpler semiclassical treatment and incorporate the spontaneous emission noise in the single-mode rate equations as Langevin noise functions, i.e.,

$$
\begin{aligned}
& \frac{d s}{d \tau}=\gamma\left[n_{\mathrm{th}} g(n)\left(1-\varepsilon_{n} s\right) s-s+n_{\mathrm{th}} \beta n\right]+\ell_{s} \\
& \frac{d n}{d \tau}=j-g(n)\left(1-\varepsilon_{n} s\right) s-n+\ell_{n} \\
& \frac{d \Phi}{d \tau}=2 \pi \tau_{e}\left(\nu_{m 0}-\nu_{0}\right)+\frac{\alpha \gamma}{2}\left[n_{\mathrm{th}} g(n)\left(1-k_{n} s\right)-1\right]+\ell_{\Phi}
\end{aligned}
$$

with

$$
g(n)=\frac{1}{n_{\mathrm{th}}}\left(n_{\mathrm{th}} n+1-n_{\mathrm{th}}\right)
$$

where $s$ and $n$ are the normalized photon and electron densities, $\tau$ is the time normalized to the electron lifetime, $j$ is the ratio between the drive current and the threshold current, $\nu_{m 0}$ is the cavity resonance frequency, $\nu_{0}$ is the lasing frequency, $\alpha$ is the linewidth enhancement factor, $\gamma=\tau_{e} / \tau_{p}$ is the ratio between the electron and photon lifetimes, $n_{\mathrm{th}}$ is the normalized carrier density at threshold, $\varepsilon_{n}$ is the normalized gain compression factor, $\beta$ is the fraction of spontaneous emission coupled to the lasing mode; the terms $\ell_{s}, \ell_{n}$, and $\ell_{\Phi}$ represent the Langevin noise sources. The various quantities and variables are normalized in accordance with previous work [9]. The novel damping parameter $k_{n}$ is introduced to account for the influence of nonlinear phenomena upon the optical phase. This approach must be understood phenomenologically. Usually, the phase depends on the change of refractive index via carriers alone and not on the spectral hole burning directly. The incorporation of this effect through $k_{n}$ is then an important point that needs comments.

The above normalized rate equations, without the inclusion of $k_{n} s$ term in (3), have been extensively discussed in a recently published paper [10]. They lead to a term explicitly involving $\varepsilon_{n}$ in the chirp-power relation referred to as adiabatic chirp which is useful for flattening the transfer function and obtaining a better modulation efficiency as well as it may be beneficial for reduced FM distortion levels. Comparing experimental results of [12] with theroretical FM response, it appears that the chirp-power relation including adiabatic chirp may be appropriate to describe CSP (channeled-substrate planar) or TJS (transverse-junction-stripe) lasers whereas a chirp-power formula without adiabatic chirp may be more suited for buried-heterostructure $(\mathrm{BH})$ lasers. Although these devices are expected to present a smaller damping factor with respect to CSP or TJS lasers, numerical values of $\mathrm{BH}$ damping factors given in the literature are not weak enough to explain the little influence of adiabatic chirp upon the 
optical FM response of that kind of lasers. Consequently, two different chirp-power formulas, with or without adiabatic chirp, have to be used to describe the FM behavior of laser diodes depending on their structure. Such a problem must be solved by establishing a more general approach capable of describing a wide variety of lasers. This is the intention behind the introduction of the $k_{n}$-parameter.

For instant, the incorporation of the refractive index nonlinearities is phenomenological. More complete investigation on $k_{n}$ is in progress. The results will be detailed in a further paper. This formalism could be justified in the sense where nonlinear effects are expected to affect differently the optical phase with respect to photon or charge carrier densities. The numerical value of $k_{n}$ can be negligible, but can also vary over a wide range depending on the device structure. It can be obtained by fitting the measured optical FM response to the theoretical expression, in a similar way as the determination of $\varepsilon_{n}$ obtained by fitting the measured IM response with theory. The influence of $k_{n}$ will be more appreciated by referring to illustrative curves appended.

\section{A. Demodulated Signal}

Equations (1)-(3) are used without the Langevin noise terms to predict the signal characteristics. Hence, we can solve (3) with (1) by eliminating $n_{\mathrm{th}} n$. Given that the dynamic chirp is $\nu_{1}=(d \Phi / d \tau) /\left(2 \pi \tau_{e}\right)$, we obtain the following relationship with the normalized photon density as defined in [9]

$$
\begin{aligned}
\nu_{1}=\nu_{m 0}-\nu_{0}+\frac{\alpha}{4 \pi \tau_{e}} & {\left[\frac{d(\ln s)}{d \tau}+\gamma\left(\varepsilon_{n}-k_{n}\right) s\right.} \\
& \left.-\beta n_{\mathrm{th}} \gamma \frac{1}{s}+\beta k_{n} \gamma\left(n_{\mathrm{th}}-1\right)\right] .
\end{aligned}
$$

From (5), it can be noticed that if $k_{n}=\varepsilon_{n}$ then adiabatic chirp cancels out while for $k_{n}=0$ one obtains formula (2) of [10].

If the laser output is passed through an interferometer whose transmission is denoted by $T(\nu)$, the normalized output intensity is $p=s T(\nu)$, which can be solved once the analytical form of the normalized photon density is specified. In particular, under the case of sinusoidal modulation, the use of a phasor representation for the involved variables, [that is, $s=s_{0}+\left(s_{\omega} e^{i \omega \tau}+s_{\omega}^{*} e^{-i \omega \tau}\right) / 2, p=p_{0}+\left(p_{\omega} e^{i \omega \tau}+\right.$ $\left.p_{\omega}^{*} e^{-i \omega \tau}\right) / 2$, where $s_{0}$ and $p_{0}$ are, respectively, the steady state parts, the star symbol stands for the complex conjugate and $\omega$ denotes the angular frequency normalized to the inverse electron lifetime] allows to calculate the complex amplitude of the signal at the output of the MZI using (5). In so doing, one obtains the following relatively simple expression

$$
p_{\omega}=\frac{\alpha T^{(1)}}{4 \pi \tau_{e}}\left[i \omega+\gamma\left(\varepsilon_{n}-k_{n}\right) s_{0}+\frac{\beta n_{\mathrm{th}} \gamma}{s_{0}}\right] s_{\omega}+T^{(0)} s_{\omega}
$$

where $T^{(i)}=\left.(\partial / \partial \nu)^{i} T\right|_{\nu_{0}},(i=0,1, \ldots)$. The fundamental IM amplitude is given in [9], [10] and is repeated here for convenience

$$
s_{\omega}=\frac{n_{\mathrm{th}} j_{\omega}}{f(\omega)}
$$

where

$$
f(\omega)=1-\frac{\omega^{2}}{\omega_{0}^{2}}+2 \eta \frac{i \omega}{\omega_{0}}
$$

in which $\omega_{0}=\left(\gamma s_{0}\right)^{1 / 2}$ and $\eta=\left(1+s_{0}+\gamma \varepsilon_{n} s_{0}+\right.$ $\left.\beta \gamma n_{\mathrm{th}} n_{0} / s_{0}\right) /\left(2 \omega_{0}\right)$. It is worth mentioning that the $\eta$ parameter expressed in [9] and [10] does not include the spontaneous emission factor. Very often, the term related to $\beta$ can be neglected compared to the other terms, but here we keep it in case its effect becomes important.

For a laser operating high enough above threshold to an extent that the spontaneous emission into the lasing mode is weak enough, one can easily calculate the CPR of the laser without interferometer using (5). The result (normalized) gives approximately

$$
\frac{\left|4 \pi \tau_{e} s_{0} \nu_{\omega}\right|}{\left|s_{\omega}\right|}=\alpha\left(\omega^{2}+\omega_{T}^{2}\right)^{1 / 2}
$$

with

$$
\omega_{T}=\gamma n_{\mathrm{th}}\left(\varepsilon_{n}-k_{n}\right)\left(j_{0}-1\right)+\frac{\beta \gamma}{j_{0}-1} .
$$

Thus, the conventional CPR is approximately constant for modulation frequencies $f \ll f_{T}=\omega_{T} /\left(2 \pi \tau_{e}\right)$. $f_{T}$ represents a kind of transition frequency which is due to gain compression and spontaneous emission term as can be seen from relation (10). In the high modulation frequency region $\left(f \gg f_{T}\right)$, the CPR will increase linearly versus $\omega$, being given approximately by an expression proportional to $\alpha \omega$. In other words, in this region, the CPR will be a straight line whose slope is proportionally related to $\alpha$. This can be developed into a technique for measuring the $\alpha$-parameter [13], [14].

On the other hand, inspection of (9) and (10) shows that the measurement of the free-running $\mathrm{CPR}$ at low modulation frequencies may also lead to a very straightforward method for measuring the spontaneous emission factor $\beta$ if $\varepsilon_{n}-k_{n}$ is known and vice-versa.

\section{B. Noise}

The noise performance of a multichannel analog transmission system is determined by its weighted signal-to-noise ratio (SNR) at the output of the system. But, even though such a process is accurate in evaluating the signal quality, another parameter easier to measure with available test equipment is the carrier-to-noise ratio (CNR). The CNR is defined as the power of the unmodulated carrier signal to the total noise power in the information-carrying channel. This definition yields a direct relationship between SNR and CNR (in decibels) through the improvement factor IF, i.e., SNR $=$ CNR + IF [15]. The CNR improvement factor in a given SCM link depends on the particular modulation format used. For AM SCM transmission systems, no gain is provided during the demodulation process, which results in $\mathrm{IF} \simeq 0 \mathrm{~dB}$. This is the reason why for AM SCM systems the terms "SNR" and "CNR" are often used without distinction. On the other hand, for FM SCM systems considerable IF-values can be achieved which can attain several tens of decibels depending on the optical FM efficiency [15]. This explains the great reliability of FM-based 
systems, being much less sensitive to noise and distortion than AM-based systems. The advantage of AM over FM is the lower signal bandwidth (6 MHz against 30-40 MHz for video transmission), required leading to a greater channel capacity. Another advantage of AM is that, in some applications as video, millions of AM-based compatible receivers already exist in the homes, so that no extra stage of demodulation is necessary.

As outlined before, the noise that affects the CNR in an analog modulation system originates both from the transmitter itself and from the receiver used. At the transmitter, the relative-intensity noise is the major noise term generally considered. In the literature, nonlinear distortion has been sometimes treated as another inherent noise term that contributes to CNR [4]. We believe, in accordance with [5], that such an approach is inappropriate since in multichannel systems, the CSO and CTB are widely accepted measures of the distortion and they are treated separately from CNR. This allows one to separate the requirements on three parameters in particular applications such as CATV [16]. Likewise, regarding nonlinear distortion as an additional noise term would be a surplus to requirements. In SCM sytems, the root-mean-square (rms) optical modulation depth (OMD) is often evaluated by setting the end-of-line CNR equal to a specified numerical value. Using a CNR expression in which nonlinear distortion term is included may result in an overestimation of the rms OMD, thereby, leading to an underestimation of the total number of channels that can be allocated.

The importance of the receiver contribution on CNR is greatly dependent on the the type of receiver considered. For a direct-detection system employing a PIN-AMP receiver, it is often assumed that the thermal noise of the preamplifier and the shot noise of the photodiode are the main noise terms that limit the CNR. The thermal noise can be reduced if alternative types of preamplifiers are used. It can be even avoided by using APD's rather than PIN-AMP's by taking advantage of their internal gain. However, on the premise that the optical receiver must be kept less expensive in order to minimize the subscriber's equipment cost, the conventional PIN-AMP receiver must be adopted as the preferred choice. Therefore, the CNR at the receiver for a single channel can be expressed (in decibels) as

$$
\mathrm{CNR}=10 \log \left\{\frac{\mathrm{OMD}^{2}\left(r P_{r}\right)^{2}}{2 B\left[\operatorname{RON}\left(f_{c}\right)\left(r P_{r}\right)^{2}+2 e r P_{r}+\left\langle I_{a}^{2}\right\rangle\right]}\right\}
$$

where $r$ is the quantum efficiency, $P_{r}$ is the average optical power received, $B$ is the channel bandwidth, $e$ is the absolute value of the electron charge and $I_{a}$ is the preamplifier thermal noise current (typically $15 \mathrm{pA}$ ). Let us note that the expression "relative-intensity noise (RIN)" often employed to characterize inherent noise effect has been replaced here by the novel expression "relative-output noise, (RON)." We found such a term is more general and convenient to keep in mind the change in the transmitter configuration. For the following, the two terms will be used depending on whether FM demodulation is or is not applied. Another comment that can be given on formula (11) is that the RON-value at the channel central frequency $f_{c}$ is taken for the entire band. This avoids mathematical integration and is permitted since the bandwidth of the information signal is generally narrow.

Relation (11) is similar to that of conventional direct detection and thus can be used to describe both techniques with and without optical discriminator. Only the parameter values have to be changed depending on each case considered. It should be mentioned, in passing, that our model assumes that the fiber does not contribute to the noise. This assumption is correct if care is taken to minimize fiber reflections in choosing good connectors and splices in order to avoid noise generated by the system [17]. On the other hand, let us also mention that (11) is valid only for direct transmission with no stage of optical amplification used. The insertion of one or several amplifiers will introduce additional noise in the system which must be included in the calculation of CNR in modifying relation (11) as appropriate.

The further examination of (11) shows that the different noise terms will not, practically, impact upon the CNR in the same way. RON noise term is seen to involve the square of the received optical power. Because FM SCM systems are much more sensitive than AM SCM systems, although they use larger channel bandwidth, large power levels are not required at the receiving end. This indicates that the RON in FM systems will not greatly influence the final CNR. Thus, generally, in such systems the receiver contribution to CNR must be the most important, although the RON noise term cannot be neglected systematically. Conversely, for AM systems, sufficiently large optical power levels are required at the receiver to achieve acceptable CNR's. In case the received optical power is weak, for example because there are a great number of users at the receiving end, one or several inline amplifier should be equipped to improve on this. In this kind of situation, the RON noise term will become the dominant noise factor. Moreover, if an FM-to-IM interferometer is employed, the optical frequency noise will be additionally converted into intensity noise causing a CNR degradation. This will be discussed further by reference to illustrative examples appended.

To derive the noise characteristics, the random deviations of the involved variables will be denoted by $\delta s, \delta n, \delta \Phi$, $\delta \nu$ and $\delta p$ whereas the corresponding Fourier transforms will be denoted by $\hat{s}_{\omega}, \hat{n}_{\omega}, \hat{\Phi}_{\omega}, \hat{\nu}_{\omega}$ and $\hat{p}_{\omega}$ (with $\hat{\nu}_{\omega}=j f \hat{\Phi}_{\omega}$ ). Mathematically, the relative-output noise and relative-intensity noise are defined in a similar way according to $\operatorname{RON}(\omega)=$ $\left\langle\left|\hat{p}_{\omega}\right|^{2}\right\rangle / p_{0}^{2}$ and $\operatorname{RIN}(\omega)=\left\langle\left|\hat{s}_{\omega}\right|^{2}\right\rangle / s_{0}^{2}$ where the numerators denote the power spectral densities of the output random fluctuations. These definitions allow to determine the relativeoutput noise using the formula (given previously) relating the Mach-Zehnder output power and the incident optical power. The calculation then yields

$$
\operatorname{RON}(\omega)=\operatorname{RIN}(\omega)+T_{r}^{2}\left\langle\left|\hat{\nu}_{\omega}\right|^{2}\right\rangle+\frac{2}{s_{0}} T_{r} \mathcal{R}_{e}\left(\left\langle\hat{s}_{\omega} \hat{\nu}_{\omega}^{*}\right\rangle\right)
$$

where $\mathcal{R}_{e}$ denotes the real part and $T_{r}=T^{(1)} / T^{(0)}$.

Equation (12) shows that the RON is a function of the classical RIN plus a term proportional to the power spectral 
density of the optical frequency noise and a term resulting from the cross-correlation between the two noise sources. Let us observe that although formula (12) is established in the situation where an interferometer is employed, it can be viewed as general, readily allowing to describe an SCM system without MZI by arbitrary setting $T_{r}=0$. Thus, for this $T_{r^{-}}$ value, the terms "RON" and "RIN" can be used as synonyms.

Relation (12) clearly predicts the RIN characteristic to degrade if the laser output is passed through an interferometer. The importance of this degradation will be more easily appreciated through further illustrative curves. The variables in (12) can be derived from the rate equations which are firstly linearized as functions of the deviations and subsequently solved in the frequency domain using Fourier analysis. The result is

$$
\begin{aligned}
{\left[\begin{array}{c}
\hat{s}_{\omega} \\
\hat{n}_{\omega}
\end{array}\right] } & =\left[\begin{array}{cc}
j \omega+m_{11} & m_{12} \\
m_{21} & j \omega+m_{22}
\end{array}\right]^{-1}\left[\begin{array}{l}
\hat{\ell}_{s} \\
\hat{\ell}_{n}
\end{array}\right] \\
\hat{\Phi}_{\omega} & =\frac{1}{j \omega}\left(m_{31} \hat{s}_{\omega}+m_{32} \hat{n}_{\omega}+\hat{\ell}_{\Phi}\right)
\end{aligned}
$$

where $\hat{\ell}_{s}, \hat{\ell}_{n}$, and $\hat{\ell}_{\Phi}$ are the Fourier transforms of Langevin noise terms; the $m_{i j}$ 's are expressed in Appendix A as constant numbers depending on the physical parameters and the bias point of the laser.

Hence, the power spectral density of the output can be calculated once the spectral characteristics of the Langevin noise forces have been specified. It is often assumed that the three noise terms have zero mean and are delta-correlated (Markovian assumption) leading to the relationship

$$
\left\langle\hat{\ell}_{q}(\omega) \hat{\ell}_{r}^{*}\left(\omega^{\prime}\right)\right\rangle=2 d_{q r} \delta\left(\omega-\omega^{\prime}\right) \quad q, r \in\{s, n, \Phi\} .
$$

where the $d_{q r}$ 's are the normalized diffusion coefficients. Because these terms are constant numbers (frequency independent), relation (15) shows that the Langevin noise sources have a white spectrum. The $d_{q r}$ 's can be expressed by the following formulas deduced from the results of [18] given in terms of number of photons and electrons

$$
\begin{aligned}
d_{s s} & =\tau_{e} \beta \gamma n_{\mathrm{th}} n_{0} s_{0} \\
d_{n n} & =\Gamma \tau_{p} n_{\mathrm{th}} n_{0}\left(\Gamma \beta s_{0}+g_{0} \tau_{e}\right) \\
d_{\Phi \Phi} & =\frac{1}{4} \tau_{e} \beta \gamma n_{\mathrm{th}} \frac{n_{0}}{s_{0}} \\
d_{s n} & =-\tau_{e} \Gamma \beta n_{\mathrm{th}} n_{0} s_{0} \\
d_{s \Phi} & =d_{n \Phi}=0 .
\end{aligned}
$$

In (17) and (19), $\Gamma$ is the optical confinement factor whereas $g_{0}$ is the gain slope (in $\mathrm{s}^{-1}$ ).

By applying the previous definition of the relative-intensity noise together with (13) and (15), a relatively simple expression can be derived for the laser without interferometer. The result can be expressed in the form

$$
\begin{aligned}
\operatorname{RIN}(\omega)=\frac{2}{\gamma^{2} s_{0}^{4}|f(\omega)|^{2}}[ & \left(m_{22}^{2}+\omega^{2}\right) d_{s s} \\
& \left.+m_{12}^{2} d_{n n}-2 m_{12} m_{22} d_{s n}\right] .
\end{aligned}
$$

Equations (16)-(21) show how the relative-intensity noise depends on the spontaneous emission factor. It can be seen
TABLE I

PARAMETERS OF SimULATION

\begin{tabular}{ll}
\hline Parameters & Values \\
\hline$\tau_{e}(\mathrm{~ns})$ & 1 \\
$\tau_{p}(\mathrm{ps})$ & 1.21 \\
$g_{0}(\mathrm{GHz})$ & $0.56210^{-5}$ \\
$\Gamma$ & 0.3 \\
$\beta$ & $10^{-4}$ \\
$\varepsilon_{n}$ & $6.4510^{-3}$ \\
$n_{t h}$ & 1.4235 \\
$\alpha$ & 6 \\
\hline
\end{tabular}

that if the second term in the right-hand side of (17) is weak enough, the RIN- $\beta$ relation will be proportional. Another comment that can be made on (21) is related to the explicit appearance of the power transfer function in the free-running RIN expression. This predicts the RIN to exhibit a resonance phenomenon at the resonant frequency of the laser diode. This will be more easily seen by reference to illustrative curves.

\section{RESUlTS AND DISCUSSION}

This section reports illustrative examples of the model developed above. The computer simulation uses the physical parameters given in Table I, in terms of normalized quantities for some of them. The results are obtained for a bias current equal to two times the threshold current.

Fig. 1 reports the frequency response of the FM-to-IM signal when the MZI is assumed to operate in the quadrature condition (half-power transmission point). The curves are plotted for a varying value of $k_{n}$. Like IM response, they exhibit a relatively pronounced peak at the resonance frequency. The effect of the $k_{n}$-parameter is seen to be little at high-modulation frequencies but a reduction in $k_{n}$ results in an increased FM efficiency in the low frequency side. It is also seen that a reduction in $k_{n}$ leads to an important extension of the flat portion of the FM response. Thus, the inclusion of this parameter could allow the description of many types of lasers without having recourse to the more complicated modeling with the Statz-de-Mars rate equations involving nonuniform carrier and optical field distributions [12]. We can notice, in this connection, that the FM response profile for low $k_{n}$ is qualitatively close to the measured data of [12] for CSP and TJS lasers, whereas the case $k_{n}=\varepsilon_{n}$ may be more accurate for the $\mathrm{BH}$ laser.

The normalized CPR is reported in Fig. 2 as decibels versus the modulation frequency in log-cordinate. As outlined before, it is seen that the CPR without MZI exhibits a relatively constant behavior at low frequencies and is approximately linear in the high modulation frequency side. The transition frequency between the two regions is seen to increase as the parameter $k_{n}$ is reduced. At low frequencies, the parameter $k_{n}$ also affects the CPR which is seen to increase with reducing $k_{n}$. On the other hand, the CPR with MZI is approximately a straight line throughout the plot range meaning that the IM term in relation (6) is negligible. Fig. 2 also indicates that when $k_{n}=\varepsilon_{n}$ the CPR, at low frequencies, is less without the MZI than with the MZI. In that case, this finding means that the MZI cannot be employed in systems operating in the low 


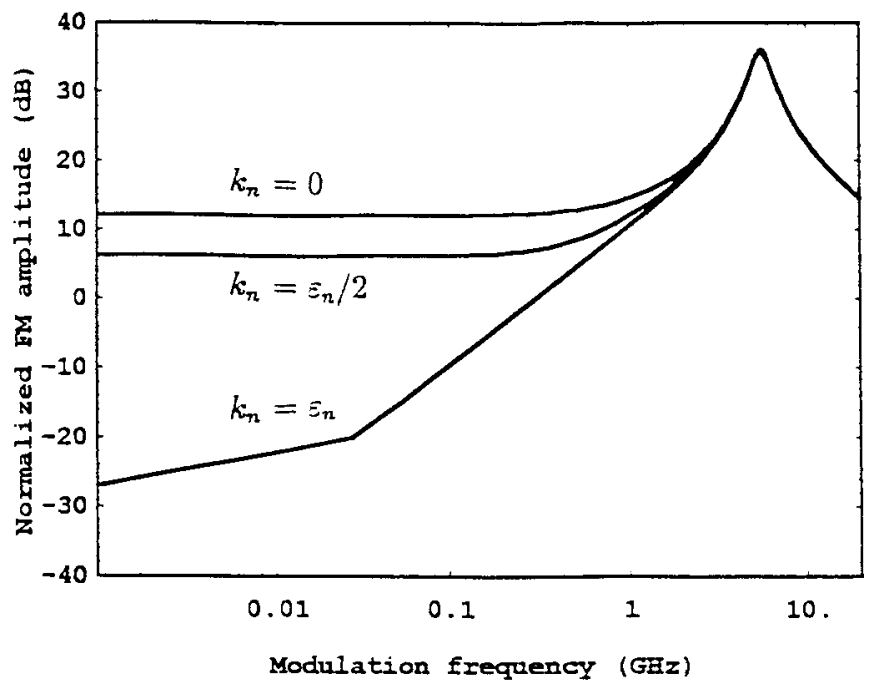

Fig. 1. Normalized frequency deviation $\left(\left|p_{\omega} / j_{\omega}\right|\right.$ without IM term) versus modulation frequency with $k_{n}$ as a parameter. The MZI was assumed to be operated in the quadrature condition.

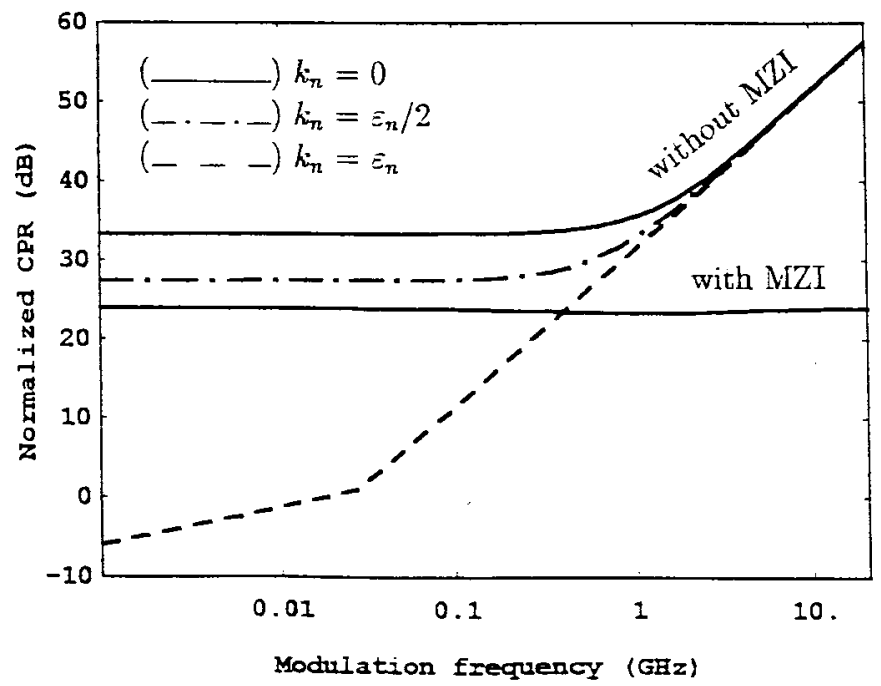

Fig. 2. Normalized CPR $\left(\left|4 \pi \tau_{e} s_{0} \nu_{\omega} / p_{\omega}\right|\right)$ as a function of the modulation frequency comparing the direct IM case to the use of a MZI operating in quadrature. In the former case, $k_{n}$ is taken as a parameter.

frequency area, such as CATV applications. Another relevant result that can be derived from Fig. 2 is that at $k_{n}=0$ the CPR reduces significantly when the laser is followed by an FM-toIM MZI operated in quadrature. We can note a reduction in CPR of more than $15 \mathrm{~dB}$ all along the plot range. It can be observed that the reduction in CPR is much more important in the high-frequency region. At $4 \mathrm{GHz}$, for example, the reported curve predicts a reduction in CPR of more than $40 \mathrm{~dB}$.

These observations express well the interest of introducing the parameter $k_{n}$. Given that a flat FM response is required for FM applications, the noise characteristics have been computed using $k_{n}=0$. Hence, Fig. 3 plots the RON as decibels versus frequency. The classical direct IM case is shown in the same figure together with the result of using a MZI. The relativeintensity noise is observed to exhibit a constant behavior in the low frequency side and is relatively low (below -150

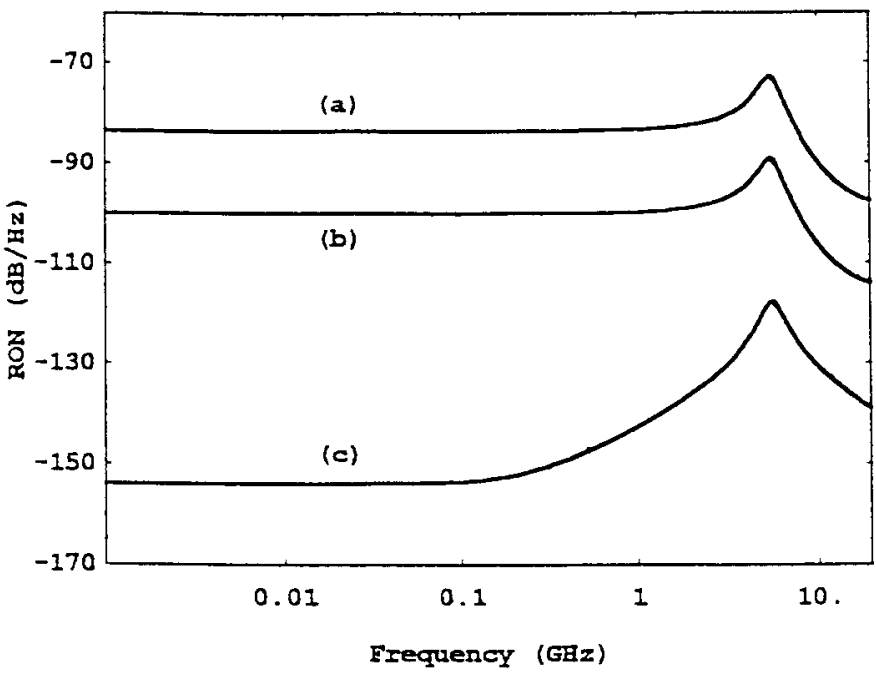

Fig. 3. Relative-output noise spectrum showing the influence of the Mach-Zehnder interferometer: (a) with an MZI for $T^{(0)}=0.022$, $T^{(1)}=0.228 \mathrm{~ns}$; (b) with an MZI operating in quadrature $\left[T^{(0)}=0.5\right.$, $\left.T^{(1)}=0.785 \mathrm{~ns}\right]$; and (c) without MZI.

$\mathrm{dB} / \mathrm{Hz}$ ). It further moves gradually to larger values above 0.1 $\mathrm{GHz}$ and attains a maximum at the resonant frequency of the transfer function. These features have been qualitatively shown in prior studies [18]. A resonance peak also appears in the relative-output noise spectrum but the peak height is much less pronounced. Besides, it is also seen that the flat portion of the curve is considerably extended, increasing from about 0.3 to $1 \mathrm{GHz}$. Unfortunately, inspection of Fig. 3 shows notably increased levels of noise when an MZI is employed. This increase is seen to be more important in the low frequency region but is generally significant all along the plot range for the two sets of MZI parameters considered. In the quadrature operation case, the relative-output noise increases more than $30 \mathrm{~dB}$ at frequencies lower than the resonance frequency. The amount of increase in RON is larger for the second operating point considered for the FM demodulator. In all cases, these results show the importance of frequency noise as dominant contribution to RON when the laser output is passed through a Mach-Zehnder. To verify this, we have simulated the same curves neglecting the direct IM noise terms. We found similar results without any significant shift in the levels at a given frequency. This suggests that CNR at large received powers the detection will be essentially limited by relative-output noise rather than shot noise. Then, even if the analysis presented in [8] is relevant, the assumption that shot noise would provide the most dominant effect is invalid. The present theory should be confirmed by experiment. Shot noise may effect may be important only for conventional CATV systems that operate below $1 \mathrm{GHz}$.

The impact of RON on CNR is shown in Fig. 4 where the carrier-to-noise ratio is reported as a function of the average received optical power, for a channel of bandwidth $B=4$ $\mathrm{MHz}$ and centered aroung $4 \mathrm{GHz}$. The classical direct IM case is also shown for comparison. The curves are plotted for the same and constant optical modulation depth of $10 \%$. The thermal noise effect appears in the low power side where the 


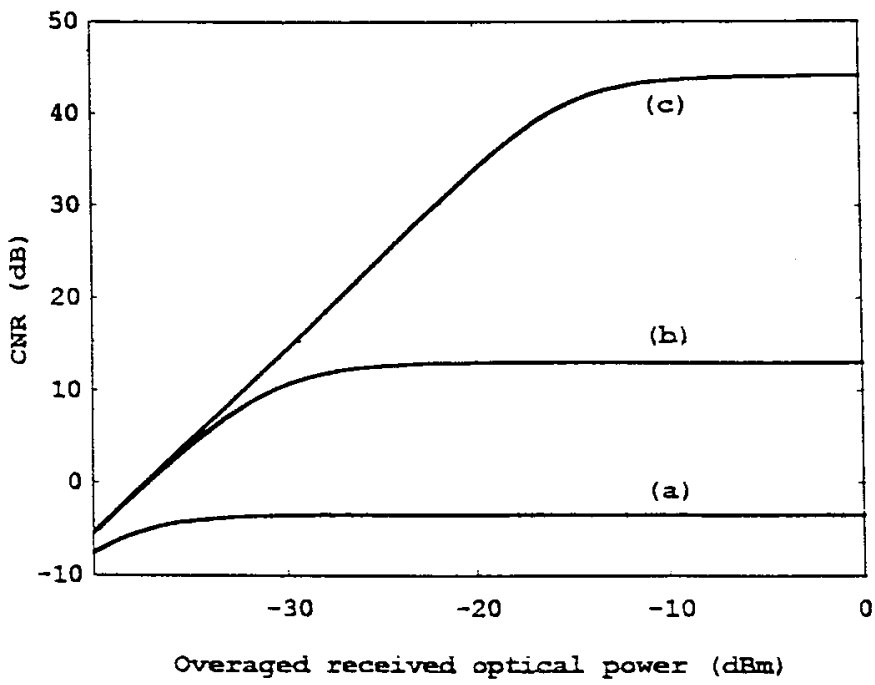

Fig. 4. Carrier-to-noise ratio as a function of the overaged received optical power at $f_{c}=4 \mathrm{GHz}$ for a channel bandwidth $B=4 \mathrm{MHz}$. The curves symbols (a), (b), and (c) have the same meaning as in Fig. 3.

CNR is seen to increase linearly. It further rises gradually and tends toward saturation. The more important feature that can be deduced from Fig. 4 is the large decrease in CNR in the high power side due to frequency noise. At $0 \mathrm{dBm}$, for example, the reduction in CNR is more than $30 \mathrm{~dB}$ in the quatrature case. The CNR even falls below $0 \mathrm{~dB}$ for the second MZI operating point considered [Fig. 4(a)]. These amounts of reduction are unacceptable for most SCM systems, even at the benefit of improved signal linearity. This result may restrict the present technique to sensitive systems only, such as those using FM signals. Because these systems need very low received powers, the CNR is not prone to degradation due to RON. On the other hand, since the FM signal does not suffer as much from the effects of distortion, the use of a MZI will allow to increase the total optical modulation depth and thus, to greatly increase the loss budget of the link under consideration [19].

Returning to the AM systems, it should be mentioned that, even though the CNR is predicted to considerably degrade, we cannot rule out the possibility of using the FM demodulation technique in such systems. Alternative solutions can be searched out to overcome the CNR impairment. One method is to find a compromise between distortion and noise by increasing the optical modulation depth. But given the high level of reduction in CNR, such a solution will not most probably suffice. For example, to completely restore the CNR to its value without interferometer at a received power of 0 $\mathrm{dBm}$, the optical modulation depth has to be increased to more than $300 \%$, which is unacceptable. Continued research is directed on technical alternatives for overcoming this constraint imposed by noise. The results will be detailed in a future paper.

\section{CONCLUSION}

A theoretical analysis of the performance of the direct optical FM demodulation technique has been carried out. We have first shown that the classical chirp-to-power ratio can be substantially reduced. Unfortunately, it is also emerged that that the direct modulation of a laser followed by an asymmetrical MZI would lead to a nonnegligible increase in RIN level which results from the conversion of the optical frequency noise into intensity noise. Consequently, the noise performance of the system is expected to degrade significantly at large received optical powers. Just as noise and distortion are known to be related inversely in regards to optical modulation depth, this result does illustrate another tradeoff between the same parameters in regards to the MZI operation conditions. The use of an MZI will indeed improve the distortion characteristics as previously shown in [9] and [10], but the extra optical frequency noise will impair the carrier-to-noise ratio available. Although experimental verification is required, this conclusion predicts a restriction in the possible implemention of the technique even though signal linearity is improved. To extend the potential applications, it seems necessary to have recourse to noise performance improvement alternatives. This question will be treated in a further study.

\section{APPENDIX}

The elements $m_{i j}$ in (11) are given by

$$
\begin{aligned}
& m_{11}=-\gamma\left[\left(n_{\mathrm{th}} n_{0}+1-n_{\mathrm{th}}\right)\left(1-2 \varepsilon_{n} s_{0}\right)-1\right] \\
& m_{12}=-\gamma n_{\mathrm{th}}\left[s_{0}\left(1-\varepsilon_{n} s_{0}\right)+\beta\right] \\
& m_{21}=\frac{1}{n_{\mathrm{th}}}\left(n_{\mathrm{th}} n_{0}+1-n_{\mathrm{th}}\right)\left(1-2 \varepsilon_{n} s_{0}\right) \\
& m_{22}=1+s_{0}\left(1-\varepsilon_{n} s_{0}\right) \\
& m_{31}=-\frac{1}{2} k_{n} \alpha \gamma\left(n_{\mathrm{th}} n_{0}+1-n_{\mathrm{th}}\right) \\
& m_{32}=\frac{1}{2} \alpha n_{\mathrm{th}} \gamma\left(1-k_{n} s_{0}\right)
\end{aligned}
$$

where $s_{0}, n_{0}$ denote the steady state average values of the variables.

\section{ACKNOWLEDGMENT}

The author would like to thank Prof. Ir. G. D. Khoe, Ir. H. de Waardt, and Dr. Ir. H. P. A. van den Boom from COBRA for their comments and helpful discussion.

\section{REFERENCES}

[1] R. Olshansky, V. A. Lanzisera, and P. M. Hill, "Subcarrier multiplexed lightwave systems for broadband distribution," J. Lightwave Technol., vol. 7, pp. 1329-1342, 1989.

[2] T. E. Darcie, R. S. Tucker, and G. J. Sullivan, "Intermodulation and harmonic distortion in InGaAsP lasers," Electron. Lett., vol. 21, pp. 665-666, 1985, and errata Vol. 22, p. 619, 1986.

[3] J. Wang, M. K. Haldar, and F. V. C. Mendis, "Formula for two-carrier third-order intermodulation distortion in semiconductor laser diodes," Electron. Lett., vol. 29, pp. 1341-1343, 1993.

[4] K. Alameh and R. A. Minasian, "Ultimate limits of subcarrier multiplexed lightwave transmission," Electron. Lett., vol. 27, pp. 1260-1262, 1991.

[5] Q. Shi, R. S. Burroughs, and D. Lewis, "An alternative model for laser clipping-induced nonlinear distortion for analog lightwave CATV systems," IEEE Photon. Technol. Lett., vol. 4, pp. 784-787, 1992.

[6] R. B. Childs and V. A. O'Byrne, "Predistortion linearization of directly modulated DFB lasers and external modulators for AM video transmission," in Tech. Dig. Optical Fiber Commun. Conf., San Francisco, CA, 1990, paper VH6.

[7] L. S. Fock and R. S. Tucker, "Simultaneous reduction of intensity noise and distortion in semiconductor lasers by feedforward compensation," Electron. Lett., vol. 27, no. 14, pp. 1297-1298, 1991. 
[8] S. L. Woodward, "Lightwave CATV systems using frequency-modulated laser and interferometer," Electron. Lett., vol. 25, pp. 1665-1666, 1989.

[9] G. Yabre and J. Le Bihan, "Intensity modulation technique using a frequency-modulated semiconductor laser and an interferometer," $J$. Lightwave Technol., vol. 13, pp. 2093-2098, 1995.

[10] G. Yabre, "Improved direct-modulation characteristics of a semiconductor laser by FM/IM conversion through an interferometer," J. Lightwave Technol., vol. 14, pp. 2135-2140, 1996.

[11] "Effects of relatively strong light injection on the chirp-to-power ratio and the $3 \mathrm{~dB}$ bandwidth of directly modulated semiconductor lasers," J. Lightwave Technol., vol. 14, pp. 2367-2373, 1996.

[12] S. Kobayashi, Y. Yamamoto, M. Ito, and T. Kimura, "Direct frequency modulation in AlGaAs semiconductor lasers," IEEE J. Quantum Electron., vol. QE-18, pp. 582-595, 1982.

[13] T. L. Koch and J. E. Bowers, "Nature of wavelength chirping in directly modulated semiconductor lasers," Electron. Lett., vol. 20, pp. 1038-1040, 1984

[14] J. E. Bowers, W. T. Tsang, T. L. Koch, N. A. Olsson, and R. A. Logan, "Microwave intensity and frequency modulation of hetero-epitaxialridge-overgrown distributed-feedback lasers," Appl. Phys. Lett., vol. 46, pp. 233-235, 1985.

[15] M. J. Pettitt, "Use of optical amplifiers to increase distribution capacity of optical analogue video systems," Inst. Elect. Eng. Proc.-Part J., 1993, vol. 140, pp. 404-410.

[16] T. E. Darcie and G. E. Bodeep, "Lightwave subcarrier CATV transmission systems," IEEE Trans. Microwave Theory Tech., vol. 38, pp. 524-533, 1990.
[17] T. E. Darcie, G. E. Bodeep, and A. A. M. Saleh, "Fiber-reflectioninduced impairments in lightwave AM-VSB CATV systems," J. Lightwave Technol., vol. 9, pp. 991-995, 1991.

[18] G. P. Agrawal and N. K. Dutta, Long-Wavelength Semiconductor Lasers. New York: Van Nostrand Reinhold, 1986.

[19] P. A. Rosher, S. C. Fenning, M. J. Ramsay, and F. V. C. Mendis, "Broadband video distribution over passive optical network using subcarrier multiplexing techniques," Electron. Lett., Vol. 2, pp. 115-117, 1989.

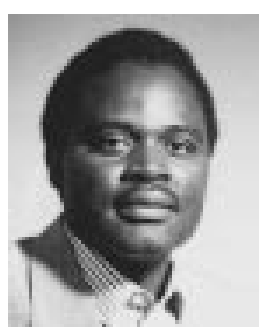

Gnitabouré Yabre (M'95) was born in BoutayaZabré, Burkina Faso, in 1962. He received the DEA degree in electronics and the Doctorate degree in optronics both from the University of Brest, France, in 1989 and 1993, respectively.

From 1989 to 1995, he worked in the RESO Laboratory at the "Ecole Nationale d'Ingénieurs de Brest," France. He is now with COBRA, Interuniversity Research Institute, Eindhoven University of Technology, The Netherlands. Previously, at "Ecole Nationale d'Ingénieurs de Brest," his research activities was focused on semiconductor laser nonlinearities, linearization techniques, optical communication systems, and networks. He is currently working mostly on polymer optical fibers for application as transmission media. 